\title{
Collaborative care may improve depression management in older adults
}

Unutzer J, Katon W,Callahan CM et al. Collaborative care management of late-life depression in the primary care setting: a randomized controlled trial.JAMA 2002 Dec;288:2836-45.

\section{QUESTION: Does collaborative care management improve late life depression?}

\section{Design}

Randomised trial with allocation concealment. Outcome assessors were blind to treatment allocation.

\section{Setting}

18 primary care clinics from 8 healthcare organisations in 5 US states; July 1999-August 2001.

\section{Participants}

1801 people aged at least 60 years (mean 71 years) with major depression (17\%), dysthymic disorder (30\%), or both (53\%). $65 \%$ were women; $23 \%$ were from an ethnic minority. Exclusion criteria were drinking problems; history of psychosis or bipolar disorder; concurrent treatment by psychiatrist; severe cognitive impairment; and acute suicide risk.

\section{Intervention}

Improving Mood Promoting Access to Collaborative Treatment (IMPACT) programme $(\mathrm{n}=906)$ or usual care $(\mathrm{n}=895)$. The IMPACT group received a video and booklet about late life depression and were encouraged to attend a consultation with a depression care manager at their primary care practice. Care managers were trained nurses or psychologists. For up to 1 year, the IMPACT group had access to a depression care manager who offered education, care management and antidepressant management, worked with the primary care physician and was supervised by a psychiatrist.

\section{Main outcome measures}

Depressive symptoms, depression treatments, satisfaction with care, functional impairment, and quality of life were measured at baseline and at 3, 6, and 12 months.

\section{Main results}

At 12 months, $45 \%$ of the IMPACT collaborative care group and $19 \%$ of the usual care group had a $50 \%$ or

Outcomes for older adults receiving collaborative care or usual care for depression

\begin{tabular}{lll}
12 month outcomes & $\begin{array}{c}\text { Odds ratio: collaborative } v \\
\text { usual care }\end{array}$ & $95 \% \mathrm{Cl}$ \\
$\begin{array}{l}50 \% \text { or greater reduction in } \\
\text { depressive symptoms from } \\
\text { baseline }\end{array}$ & 3.5 & 2.7 to 4.4 \\
\hline $\begin{array}{l}\text { Additional treatment for } \\
\text { depression }\end{array}$ & 3.0 & 2.3 to 3.8 \\
\hline Satisfaction with care & 3.4 & 2.7 to 4.3 \\
\hline
\end{tabular}

greater reduction in depressive symptoms from baseline $(\mathrm{P}<0.05)$. The IMPACT group also had higher rates of depression treatment and greater satisfaction with depression care compared with usual care (see table). The IMPACT group had reduced depression severity, less functional impairment, and improved quality of life (all $\mathrm{P}<0.001)$.

\section{Conclusions}

The IMPACT collaborative care model appears more effective than usual care for reducing depressive symptoms in later life.

\section{COMMENTARY}

This sizeable trial builds upon previous studies of collaborative care and chronic disease management. The authors suggest that this collaborative, stepped care management intervention is more effective than usual care for depression in a wide range of people and several types of medical care organisations. The findings have important implications for clinical practice and challenges for current policy.

Several randomised trials have found improved patient outcomes in depression from various components of chronic disease management compared with usual care. Although this study focused on older people, the totality of evidence suggests that usual care for major depression or dysthymia in primary care is no longer acceptable for any age group. Beyond screening or increased case detection, all primary care providers and practice administrators should examine how they can improve depression management. Depending on the size and resources of a practice, at least some of the care management components of this intervention could be implemented. For the mental health worker, this study emphasises the importance of seeking out and being involved in consultative and supervisory roles to more effectively serve a larger number of patients.

Investigators should not be complacent that interventions of this nature will improve depression management. The number of people achieving true remission remains unacceptably small, attesting to the chronic nature of major depression and dysthymia.

For government and organisational policy makers, the results of this study mandate the need to offer fair and reasonable reimbursement for care management services and for telephone or in person supervision by mental health professionals serving primary care providers.

Thomas E Oxman, MD Department of Psychiatry Dartmouth Medical School Lebanon, NH, USA 